\title{
A Programmable Synthesis of Diverse Terpene Architectures from Phenols
}

Farbod Salahi, ${ }^{1}$ Chengbo Yao, ${ }^{2}$ Jack R. Norton, ${ }^{2}$ and Scott A. Snyder*1

${ }^{1}$ Department of Chemistry, University of Chicago, 5735 S. Ellis Avenue, Chicago, IL 60615

${ }^{2}$ Department of Chemistry, Columbia University, 3000 Broadway, New York, NY 10027

Over millennia, Nature has evolved exquisite synthetic pathways that combine isoprenes into chains, folds them into carbocycles, and then oxidizes/rearranges them into vast complexity $(>50,000$ members). While laboratory chemical synthesis can sometimes emulate this process, room exists for additional approaches that can also programmably lead to molecular diversity. Here we show that from simple phenols using 1) prenylation, 2) dearomatization/prenyl migration, and 3) epoxidation/radical cyclization, we can predictably, reliably, and expeditiously make polycyclic terpene frameworks; critically, step three uses the first cooperative bimetallic catalyst to effect cyclization of epoxy enones under $\mathrm{H}_{2}$. Indeed, our approach has led to the stereocontrolled formation of bicyclic, linear, angular, clovane, and propellane-based architectures with functional groups that allow further manipulation; for example, these motifs can be repurposed for ring contractions. Of note, several formal total syntheses have been achieved in routes that are as concise as, and often shorter than, previous efforts.

Nature has evolved highly efficient pathways to fashion diverse terpenes from common building blocks through unified synthetic approaches based on cyclase/oxidase logic. ${ }^{1-4}$ Given that model for efficient diversity generation, we became interested in developing similarly effective sequences that use components other than isoprene units and that involve different chemical transformations.

Specifically, as shown in Figure 1, we hoped that differentially patterned phenol derivatives 1-3 could afford distinct terpene architectures in a systematic and predictable manner. ${ }^{5}$ Indeed, if these phenols were furnished with an isoprene unit in the form of a prenyl sidechain, we hoped that the known, but rarely employed, para-selective Claisen rearrangement ${ }^{6}$ could afford dearomatized materials such as $\mathbf{4 , 5}$, and $\mathbf{7}$. And, if that rearrangement were conducted under suitable reductive conditions, structures such as 6 and 8 could also result in the same pot, with their more accessible, less-substituted, alkenes no longer present.

From here, a site-specific epoxidation of the exocyclic, trisubstituted alkenes in 4-8 would allow access to a reactive handle by which to utilize Giese chemistry7 to generate 
tertiary carbon-centered radicals. ${ }^{8}$ These radicals should add to the $\beta$ carbons of the enones in a 5-exo-trig manner and forge the new five-membered rings in 9-14. These rings would contain both a gem-dimethyl group and a secondary alcohol with contiguous tertiary and/or quaternary stereogenic centers that are challenging to fashion with other methods. ${ }^{9}$ For dienones (like 5 and 7), the cyclization should occur selectively at the more accessible of the two alkenes, while in those substrates where one enone has already been reduced (like 6 and 8 ), cyclization onto the remaining enone should be achievable despite the steric encumbrance. While examples of such cyclizations are well documented with simple enones, ${ }^{10,11}$ and illustrated by our recent synthesis of the conidiogenones, ${ }^{12}$ it was unclear how well they would work with dienones where rearrangements ${ }^{13}$ could restore aromaticity prior to cyclization. In addition, these cyclizations typically require superstoichiometric quantities of metal species to achieve, with the most common promoter being the Nugent-RajanBabu reagent; ${ }^{14}$ as such, we hoped to develop a catalytic version, especially if the few procedures of this type known ${ }^{15}$ could not rise to the occasion.

Finally, if needed, we expected that the core six-membered ring, with its strategically positioned ketone and neighboring methylene derived from a carbon of the original phenol, could be induced to undergo ring contraction (via a Wolff or Favorskii rearrangement, for example). ${ }^{12,16}$ We would thus have controlled, programmable, and divergent ${ }^{17}$ access to bicyclic, clovane, linear, angular, and propellane-type ring systems relevant to hundreds of terpenoid natural products (with 15-21 being representative cases), from a common set of starting materials and reaction processes. ${ }^{18}$

Herein, we show that this design can be realized, with key discoveries being a onepot reductive dearomatization procedure and the identification of a catalytic system that can achieve the radical cyclization with equal facility as stoichiometric promoters. The sequence has broad scope and functional group tolerance, and its power is highlighted by several formal total syntheses of natural products as well as by the preparation of natural product analogs. These syntheses proceed with stepcounts equivalent or reduced compared to those of previous efforts, many of which generated only a single terpenebased architecture. ${ }^{18}$ We believe our overall process provides important complementarity both to Nature's synthesis of terpenes, and to other recent examples of terpene polycycle synthesis via radical cyclizations. ${ }^{19-22}$

\section{Results and Discussion}

We began by exploring the scope of the initial dearomatization using bulky aluminum Lewis acids such as MABR and MAD (22 and 23, respectively, Figure 2) as developed by Yamamoto.6,23,24 This reaction has been rarely employed despite its potential; to the best of our knowledge there has been only one use (with one substrate), in natural product synthesis, racemically by Danishefsky ${ }^{25}$ and asymmetrically by Lawrence. ${ }^{26}$ As such, we have more fully explored the scope of the dearomatization with $\mathbf{2 2}$ and $\mathbf{2 3}$, and as denoted below, have developed a related reaction, a one-pot reductive dearomatization, in which one of the enones is reduced to afford compounds like 6 and $\mathbf{8}$. This reductive dearomatization is complementary to existing oxidative allylative dearomatizations of 
phenols as mediated by hypervalent iodine reagents, such as phenyliodine diacetate (PIDA); ${ }^{27}$ the major disadvantage of this method is poor functional group compatibility as well as the site-selectivity in the allylation (i.e. ortho-vs. para-selectivity).

As shown in Figure 2, in all cases examined (see SI for the synthesis of the prenylated starting materials) the para-Claisen rearrangement performed well using $\mathbf{2 2}$ as the preferred promoter. The conditions were mild enough that TBS-protected primary alcohols (34-36), TMS-protected tertiary alcohols (32), Boc-protected amines (38), and even free alcohols (46) were tolerated. Aryl halides worked as well, affording vinylic halide products $\mathbf{3 0}$ and $\mathbf{3 1}$, as did the 4-membered ring of $\mathbf{3 2}$ (which can potentially afford a spirocyclic system after a semi-pinacol rearrangement).28

In all cases, although racemic starting materials were generally used (exceptions were the precursors to $\mathbf{4 5}$ and $\mathbf{4 6}$ ), the reaction process was fully diastereoselective, with the prenyl sidechain ultimately occupying the same face as the large group of those preexisting centers. We believe that outcome is governed by minimizing any steric clash between the original chiral center within the substrate and the complexed aluminum reagent (see SI for additional schemes illustrating these outcomes). ${ }^{29}$ Whether these events proceed either via a direct and/or stepwise migration pathway remains unknown as also denoted by Yamamoto; minor amounts of aromatized side-products (of general type 26) were observed on occasion. ${ }^{6}$

While dearomatization with $\mathbf{2 2}$ or $\mathbf{2 3}$ was broad in scope, the one-pot reductive dearomatization proved, to our delight, to be achievable as well. Specifically, if we completed the initial prenyl migration at $-78^{\circ} \mathrm{C}$ and then added a stoichiometric amount of L-Selectride, we obtained selectively reduced adducts like 6, 8, 28, 37, 41, 42, 44, and 46. For unsymmetrical dienones, the more accessible alkene was reduced preferentially. Finally, certain non-prenyl substituents also migrated effectively, including the crotyl motif within 47 (albeit in a modest yield, Yamamoto has also reported one example of such a migration) $)^{6}$ and the more complex geranyl chain within 48.

With these dearomatized products in hand, we next probed our ability to fold them into an array of terpene architectures - by epoxidizing them, then opening the epoxides with $\mathrm{Ti}(\mathrm{III})$ and letting the resulting radicals add intramolecularly (5-exo-trig) to the $\beta$ carbon of an enone (top of Figure 3). For epoxidation we used $m$ CPBA, while for initial probes of the radical cyclizations we used superstoichiometric amounts of the NugentRajanBabu reagent ( 3 to 3.5 equiv as generated from $\mathrm{Cp}_{2} \mathrm{TiCl}_{2}$ and $\mathrm{Zn}$ in $\mathrm{THF}$ at $23^{\circ} \mathrm{C}$ ). ${ }^{14} \mathrm{In}$ all cases, the less encumbered $\mathrm{C}=\mathrm{C}$ bond of the dienone served as the radical acceptor and no rearrangements were observed. With a monosubstituted dienone such as $\mathbf{3 0}$, the presence of a bulky group on one of the dienone olefins at the site of cyclization effected complete regiochemical control, while more remote substitution (29) had little effect ( $\sim 1: 1$ selectivity). For those substrates that were pre-reduced, the remaining (more hindered) enone reacted smoothly to generate angular structure $\mathbf{1 2}$ as well as the highly congested propellane architectures $14,58,59$, and 61 possessing four to five contiguous tertiary and/or quaternary centers. Overall, there was good diastereocontrol in many cases (the major diastereomer is drawn, with the configuration confirmed by X-ray crystallography in many cases). As shown graphically in the Supporting Information section, we believe the 
origin of this diastereoselectivity is different for symmetrical and unsymmetrical dienones. For the former, radical cyclization appears to be the diastereo-determining step, with the major diastereomer resulting via a transition state that minimizes syn-pentane interactions. For the latter, the diastereoselectivity appears to reflect the initial diastereomeric ratio obtained from the epoxidation step, one dictated by a minimization of steric hindrance of the reactive conformer through $\mathrm{A}^{1,3}$ strain. $^{30}$

Outside of this stereochemical control feature, critical more generally to the overall design is the ability to differentiate radical stability from the epoxide. For example, similar substituents on the two epoxide carbons (e.g., in the crotyl substrate 47) gave a low yield of the desired cyclized product (63) with significant amounts of its rearomatized counterpart 64. Of note, the 2,2-disubstituted terminal olefin 49 (not prepared by dearomatization, see SI for details) led to the formation of a four-membered ring product via the more stable tertiary carbon-centered radical. Finally, although not obtained via a direct prenyl migration pathway, substrate $\mathbf{5 0}$ highlights that six-membered rings can result as well. Many of the products in Figure 3 were obtained on gram scale, illustrating the facility of the chemistry and the potential of the developed sequence for complex molecule synthesis (vide infra).

We then sought to develop a catalytic variant of the stoichiometric Ti(III) reaction in Figure 3. As one reflection of the challenge of effecting such chemistry in these systems, efforts using other approaches to catalysis as developed by Gansäuer (entries 1 and 2 in Figure $4 \mathrm{a})^{15}$ were unsuccessful. Pleasingly, however, the use of $10 \mathrm{~mol} \%$ each of $\mathrm{CpCr}(\mathrm{CO})_{3} \mathrm{H}(73), \mathrm{NaCpCr}(\mathrm{CO})_{3}$, and $\mathrm{Cp}_{2} \mathrm{Ti}(\mathrm{OMs})_{2}$ in benzene, at $70{ }^{\circ} \mathrm{C}, 31$ provided an $\sim 1: 1$ mixture of the desired product $\mathbf{5 1}$ and an undrawn epoxide-derived $O$-cyclized product in $47 \%$ yield each (entry 3). Performing the reaction at a lower temperature $\left(50{ }^{\circ} \mathrm{C}\right)$ gave only the desired product, but the yield was low even following an extended reaction time (entries 4 and 5). Pleasingly, more dilute conditions at $70{ }^{\circ} \mathrm{C}$ afforded $\mathbf{5 1}$ in $85 \%$ isolated yield, with no appreciable amount of the $O$-cyclized material (entry 6). We believe this reaction occurs with opening of the epoxide by the Ti catalyst (Figure 4c), effecting cyclization to intermediate $\mathbf{A}$. HAT onto the oxoallylic radical of $\mathbf{A}$ can then generate $\mathbf{B}$, which can undergo protonation by $\mathrm{HCpCr}(\mathrm{CO})_{3}$ to provide the product. A bimetallic $\mathrm{Cr}-\mathrm{Ti}$ complex 72 recently synthesized by our group ${ }^{32}$ also proved to be an active catalyst, albeit in reduced yield (entry 7). The catalytic variant of this reaction has excellent scope, behaving similarly (in terms of yield and $d r$ when applicable) to the stoichiometric system in Figure 3. The catalytic reaction can also be conducted smoothly on a gram scale.

Finally, to test the power of our overall sequence in complex molecule synthesis, we targeted a number of natural products and analogs. Simple oxidation of 51, shown in Figure 5, afforded 74. This material was previously used to synthesize cispreisothapsadiene (15), ${ }^{33}$ with our stepcount to $\mathbf{7 4}$ being half that previously reported. Alternatively, Barton-McCombie deoxygenation of the alcohol in 51, followed by a diazo transfer and photochemical Wolff rearrangement, gave a ring contracted ester which upon isomerization with DBU generated the $\alpha, \beta$-unsaturated ester 75. Efforts to attempt other ring contractions, ${ }^{16}$ such as methods using $\mathrm{Ti}\left(\mathrm{NO}_{3}\right)_{3}$ and a Favorskii reaction using PIDA, were not fruitful. Compound $\mathbf{7 5}$ could then be converted in two steps via a Weinreb amide 
into 76, a compound that Paquette had shown could be easily converted into $\Delta^{9(12)}$ capnellene (77). ${ }^{34}$ Similarly, the deoxygenation of $\mathbf{6 6}$ completed a 6-step synthesis of 78, a material previously prepared in nine steps during a total synthesis of ambrox (79). ${ }^{35}$ Finally, prenyl migration with $\mathbf{8 0}$ under our reductive conditions, and subsequent epoxide formation, afforded $\mathbf{8 1}$ - giving us access to this intermediate used for isocyanoneopupukeanane (82) in half the earlier stepcount. ${ }^{36}$

As shown in Figure 6, this general strategy also offers expedient access to clovane and propellane-type natural products and their analogs. For the former, dehydration of $\mathbf{5 5}$ followed by conjugate reduction and methylation gave $\mathbf{8 3}$, where we expected that the new alkene could facilitate eventual access to targets such as $\mathbf{8 5}$ and $\mathbf{8 6}$. Of these steps, enone reduction proved the most problematic due to the presence of the adjoining quaternary center; indeed, efforts using solely L-Selectride or the "hot" Stryker's reagent ${ }^{37}$ gave 1,2reduction or low yield of desired product, respectively. Pleasingly, the conditions developed for our reductive dearomatization (i.e., MAD and L-Selectride), effected the desired conjugate reduction in good yield. Finally, the pendant OTBS group within 83, following silyl cleavage and Dess-Martin periodinane oxidation, permitted an aldol closure to generate the final ring of $\mathbf{8 4}$, thereby affording a fully decorated skeleton of the clovane natural product family. For the propellane natural products (Figure 6), differential protection of the hydroxyls of $\mathbf{5 7}$ and 58, followed by diazoketone synthesis/Wolff rearrangement, afforded the desired ring-contracted esters as a mixture of regio- and stereoisomers (one confirmed by X-ray; see SI). Nucleophilic demethylation, nickelcatalyzed decarboxylation, ${ }^{38}$ and Dess-Martin periodinane-mediated oxidation gave 91 thereby affording formal access to epi-modhephene (92). ${ }^{39}$ of note, we obtained intermediate 91 in 14 steps, a level of efficiency that compares favorably to the length of the previous route (20 steps); it was also obtained in reasonable quantity (170 $\mathrm{mg})$. An added deprotection step afforded 93, a subtly different variant of an intermediate successfully advanced to the dichrocephones (94 and 95) by Christmann. ${ }^{40}$

\section{Conclusion}

This work provides a programmable, consistent, but ultimately divergent sequence to access various terpene-based architectures within the triquinane family of natural products using precursors distinct from those employed by Nature. To reach that objective, we offer significant extensions of previously developed methods, and combine them in novel ways with unique condition sets to achieve the final sequences. Of particular significance are a one-pot dearomatization/reduction protocol that directly affords enones and an effective, cooperative catalytic system for the cyclization of epoxy enones with $\mathrm{H}_{2}$ as the terminal reductant. At the end, a range of complex architectures is accessible, most in shorter stepcounts than previous efforts and many in gram scale quantities. Prenyl groups are part of our process, just as Nature often uses isoprene units, though the overall design is clearly distinct. Time will tell if there are advantages to our approach over the pathways that Nature has developed, though we believe the added efficiency in our formal total syntheses indicates that optimism is warranted. 


\section{Acknowledgements}

We thank Alexander Filatov and Kate Jesse (University of Chicago) for X-ray analysis of our crystalline intermediates, and Josh Kurutz and C. Jin Qin for assistance with NMR and mass spectrometry, respectively (University of Chicago). This work was supported by funds from the University of Chicago and the NIH (R01-124295A).

\section{Author contributions}

S.A.S. and F.S. conceived the project, while S.A.S. and J.R.N. directed the research. F.S. designed, carried out, and analyzed all experiments of the main manuscript, except for those studies on the catalytic approach for the epoxy-enone cyclizations (which were performed by C.Y.). S.A.S. and F.S. composed the manuscript and the Supporting Information Section; all authors commented on the manuscript.

\section{Competing financial interests}

The authors declare no competing financial interests.

\section{References}

1. Christianson, D.W. Structural and Chemical Biology of Terpenoid Cyclases. Chem. Rev. 117, 11570-11648 (2017).

2. Fischbach, M., Clardy, J. One pathway, many products. Nat. Chem. Biol. 3, 353-355 (2007).

3. Harms, V., Kirschning, A. \& Dickschat, J. S. Nature-driven approaches to non-natural terpene analogues. Nat. Prod. Rep. 37, 1080-1097 (2020).

4. Kanda, Y. et al. Two-phase synthesis of taxol. J. Am. Chem. Soc. 142, 10526-10533 (2020).

5. Wildermuth, R., et al. A modular synthesis of tetracyclic meroterpenoid antibiotics. Nat. Commun. 8, 2083 (2017). https://doi.org/10.1038/s41467-01702061-7.

6. Maruoka, K., Sato, J., Banno, H. \& Yamamoto, H. Organoaluminum-promoted rearrangement of allyl phenyl ethers. Tetrahedron Lett. 31, 377-380 (1990).

7. Giese, B. Formation of CC Bonds by Addition of Free Radicals to Alkenes. Angew. Chem. Int. Ed. Engl. 22, 753-764 (1983).

8. Streuff, J. \& Gansäuer, A. Metal-Catalyzed $\beta$-Functionalization of Michael Acceptors through Reductive Radical Addition Reactions. Angew. Chem. Int. Ed. 54, 1423214242 (2015).

9. Büschleb, M. et al. Synthetic strategies toward natural products containing contiguous stereogenic quaternary carbon atoms. Angew. Chem. Int. Ed. 55, 41564186 (2016).

10. Morcillo, S. P. et al. Ti(III)-catalyzed cyclizations of ketoepoxypolyprenes: control over the number of rings and unexpected stereoselectivities. J. Am. Chem. Soc. 136, 6943-6951 (2014).

11. Lo, J. C. et al. Fe-Catalyzed C-C bond construction from olefins via radicals. J. Am. Chem. Soc. 139, 2484-2503 (2017).

12. Hu, P. et al. Quaternary-centre-guided synthesis of complex polycyclic terpenes. Nature. 569, 703-707 (2019) 
13. Xia, Z. et al. $\mathrm{Re}_{2} \mathrm{O}_{7}$ catalyzed dienone-phenol rearrangement. RSC Adv. 5, 3849938502 (2015).

14. Nugent, W. A. \& RajanBabu, T. V. Transition-metal-centered radicals in organic synthesis. Titanium(III)-induced cyclization of epoxy olefins. J. Am. Chem. Soc. 110, 8561-8562 (1988).

15. Zhang, Z., Richrath, R. B., Gansäuer, A. Merging Catalysis in Single Electron Steps with Photoredox Catalysis-Efficient and Sustainable Radical Chemistry. ACS Catal. 9, 3208-3212 (2019).

16. Silva, L. F. Construction of cyclopentyl units by ring contraction reactions. Tetrahedron, 58, 9137-9161 (2002)

17. Li, L., Chen, Z., Zhang, X. \& Jia, Y. Divergent Strategy in Natural Product Total Synthesis. Chem. Rev. 118, 3752-3832 (2018)

18. Le Bideau, F., Kousara, M., Chen, L., Wei, L. \& Dumas, F. Tricyclic sesquiterpenes from marine origin. Chem. Rev. 117, 6110-6159 (2017).

19. Hung, K., Hu, X. \& Maimone, T. J. Total synthesis of complex terpenoids employing radical cascade processes. Nat. Prod. Rep. 35, 174-202 (2018).

20. Gansäuer, A. \& Narayan, S. Titanocene-catalysed electron transfer-mediated opening of epoxides. Adv. Synth. Catal. 344, 465-475 (2002).

21. Barrero, A. F., Quílez del Moral, J. F., Sánchez, E. M. \& Arteaga, J. F. Titanocenemediated radical cyclization: an emergent method towards the synthesis of natural products. Eur. J. Org. Chem. 7, 1627-1641 (2006).

22. Justicia, J. et al. Bioinspired terpene synthesis: a radical approach. Chem. Soc. Rev. 40, 3525-3537 (2011).

23. Saito, S. \& Yamamoto, H. Efficient Conjugate Reduction of $\alpha, \beta$-Unsaturated Carbonyl Compounds by Complexation with Aluminum Tris(2,6-diphenylphenoxide). J. Org. Chem. 61, 2928-2929 (1996).

24. Saito, S. \& Yamamoto, H. Designer Lewis acid catalysts-bulky aluminium reagents for selective organic synthesis. Chem. Commun. 1585-1592 (1997).

25. Lei, X., Dai, M., Hua, Z. \& Danishefsky, S. J. Biomimetic total synthesis of tricycloillicinone and mechanistic studies toward the rearrangement of prenyl phenyl ethers. Tetrahedron Lett. 49, 6383-6385 (2008).

26. Homer, J. A. et al. Enantioselective para-Claisen Rearrangement for the Synthesis of Illicium-Derived Prenylated Phenylpropanoids. Org. Lett. 23, 3248-3252 (2021).

27. Guérard, K. C. et al. Oxidative 1,2- and 1,3-Alkyl Shift Processes: Developments and Applications in Synthesis. J. Org. Chem. 77, 2121-2133 (2012)

28. Hou, H. et al. Total Syntheses of the Tetracyclic Cyclopiane Diterpenes Conidiogenone, Conidiogenol, and Conidiogenone B. Angew. Chem. Int. Ed. 55, 44564460 (2016).

29. Maruoka, K., Banno, H, \& Yamamoto, H. Enantioselective Activation of Ethers by Chiral Organoaluminum Reagents: Application to Asymmetric Claisen

Rearrangement. Tetrahedron: Asymmetry, 2, 663-666 (1991).

30. Hoffmann, R. W. Allylic 1,3-strain as a controlling factor in stereoselective transformations. Chem. Rev, 89, 1841-1860 (1989).

31. Yao, S., Dahmen, T., Gansäuer, A. Norton, J. Anti-Markovnikov alcohols via epoxide hydrogenation through cooperative catalysis. Science 364, 764-767 (2019). 
32. Chen, J. et al. Synthesis, Characterization, and Catalytic Activity of Bimetallic Ti/Cr Complexes. Organometallics 39, 4592-4598 (2020).

33. Srikrishna, A. \& Mahesh, K. An Enantiospecific Approach to Irregular Ligusticum grayi Sesquiterpenes: Synthesis of cis-Preisothapsa-2,8(12)-diene. Synlett 17, 25372540 (2011).

34. Stevens, K. E. \& Paquette, L. A Stereocontrolled total synthesis of $( \pm)-\Delta 9(12)-$ capnellene. Tetrahedron Lett. 22, 43934396 (1981).

35. Kutney, J. P. \& Chen, Y. H. The chemistry of thujone. XVII. The synthesis of ambergris fragrances and related analogues. Can. J. Chem. 72, 1570-1581 (2011).

36. Ho, T. S. \& Jana, G. H. Total Synthesis of 9-Isocyanoneopupukeanane. J. Org. Chem. 24, 8965-8967 (1999).

37. Baker, B.A., Bošković, Ž. V., Lipshutz, B. H. (BDP)CuH: A “Hot” Stryker's Reagent for Use in Achiral Conjugate Reductions. Org. Lett. 10, 2, 289-292 (2008).

38. Qin, T. et al. Nickel-catalyzed Barton decarboxylation and Giese reactions: a practical take on classic transforms. Angew. Chem. Int. Ed. 56, 260-265 (2017).

39. Curran, D. P. \& Shen, W. Tandem transannular radical cyclizations. Total syntheses of ( \pm )-modhephene and ( \pm )-epi-modhephene. Tetrahedron 49, 755-770 (1993).

40. Schmiedel, V. M. et al. Synthesis and structure revision of dichrocephones A and B. Angew. Chem. Int. Ed. 57, 2419-2422 (2018). 

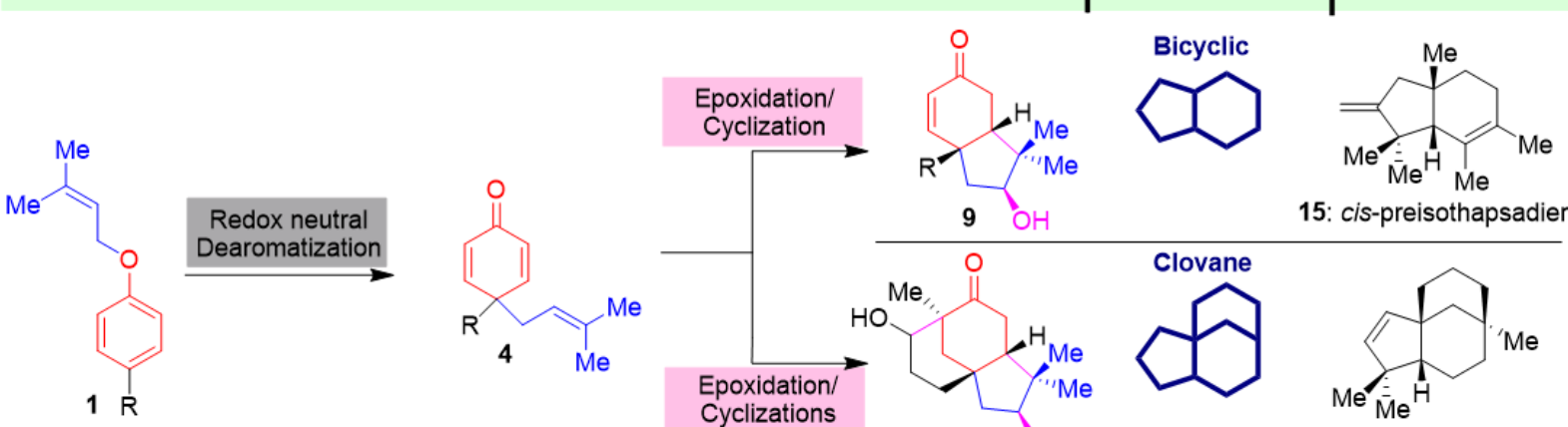

15: cis-preisothapsadiene

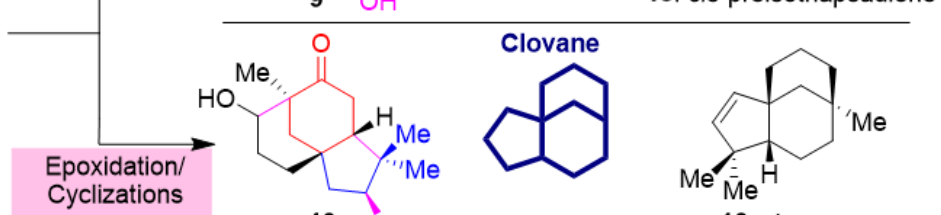

$10 \mathrm{OH}$

16: clovene

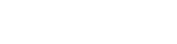
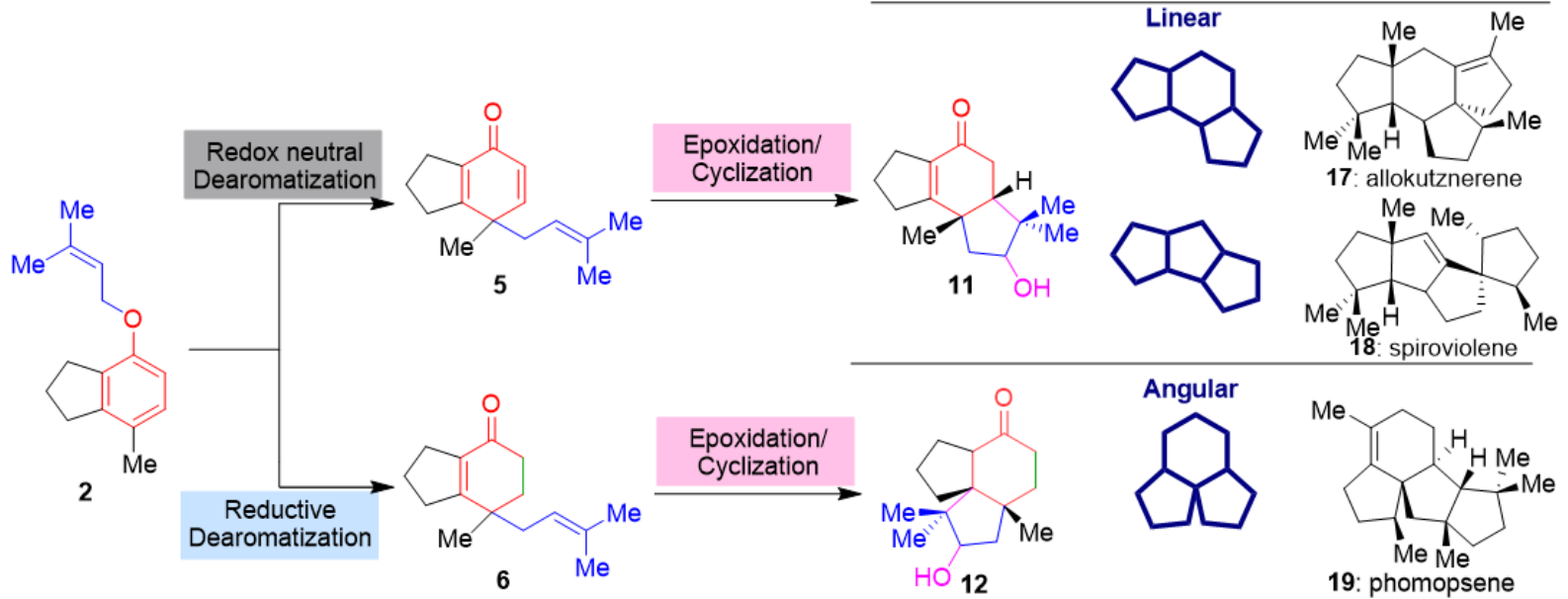

2
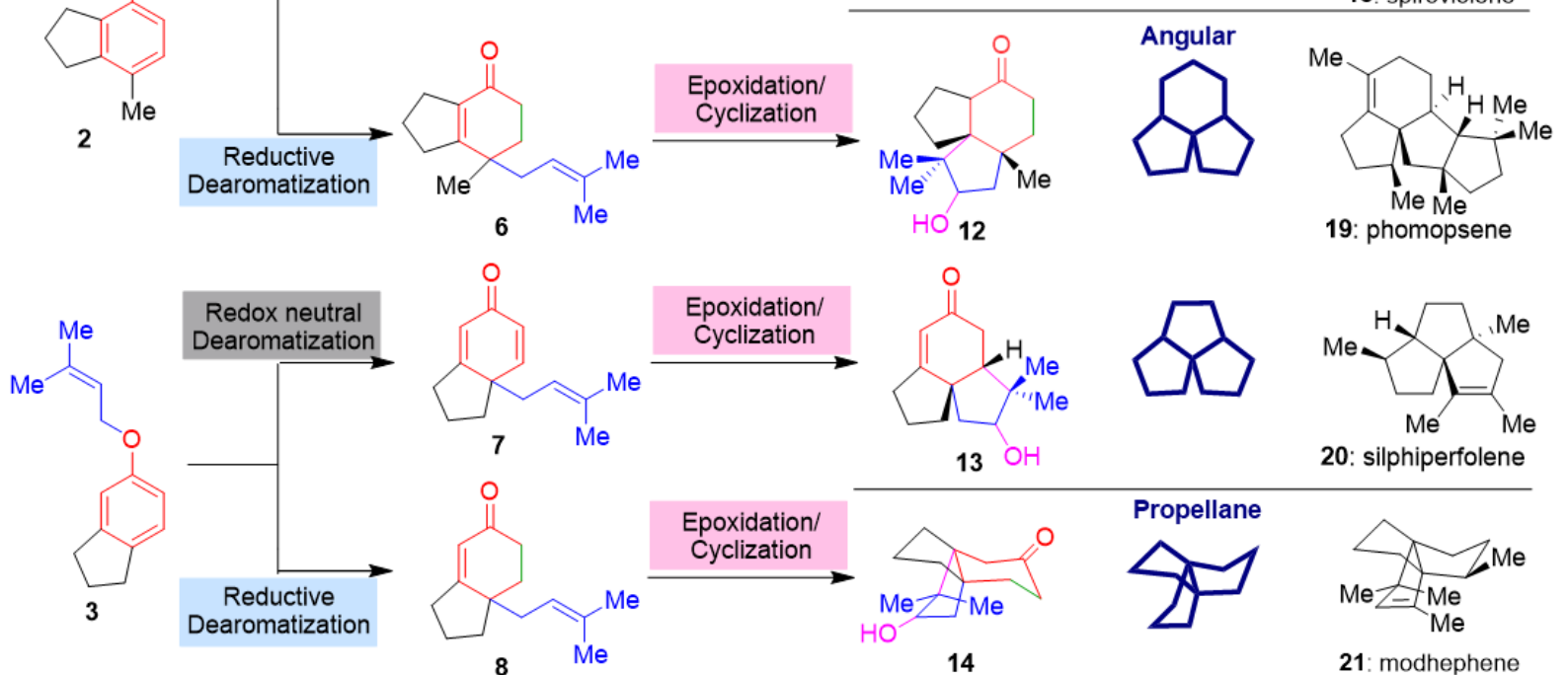

20: silphiperfolene

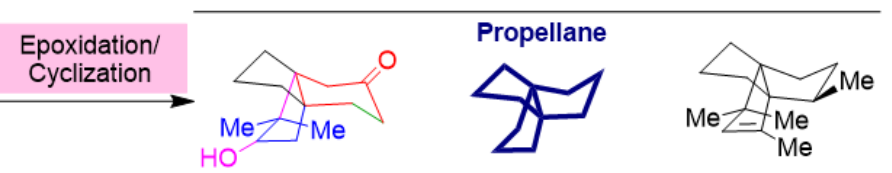

14

21: modhephene

Figure 1 | Development of a cohesive and unified strategy for terpene synthesis. Varied dearomatizations using prenyl migrations, followed by epoxidation and radical cyclization, can access numerous terpene frameworks relevant for natural product total synthesis. 


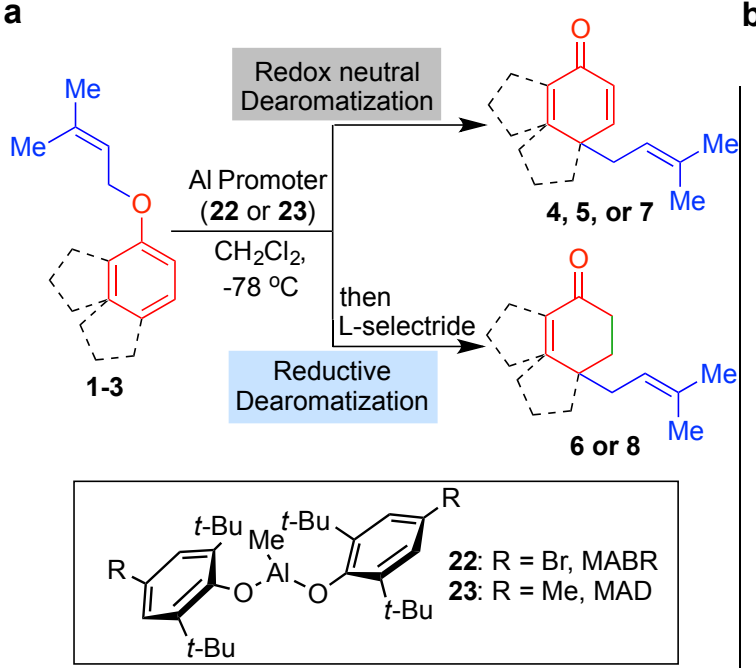

C

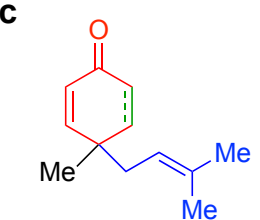

27 (76\%; gram scale)

28 (reduced, 54\%)

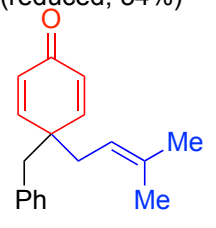

33 (78\%)

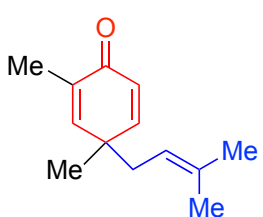

$29(83 \%)$

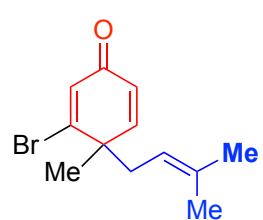

30 (51\%)
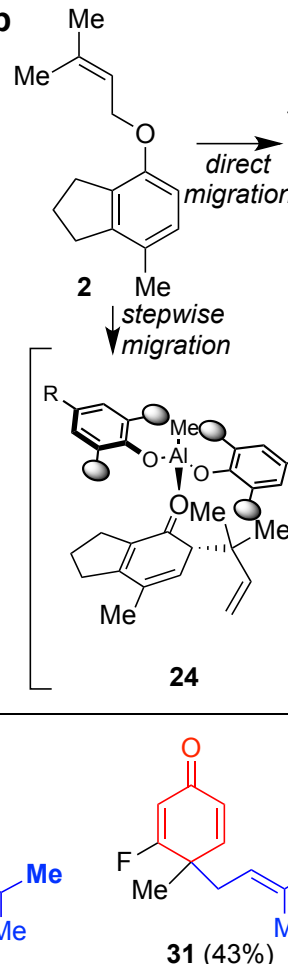

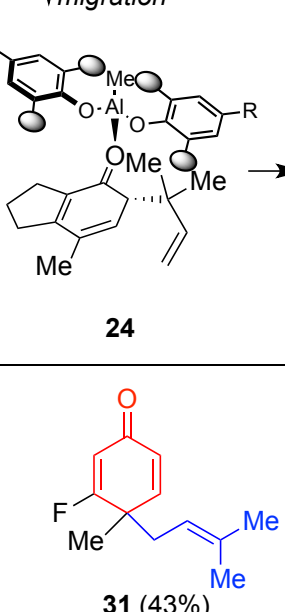

31 (43\%)
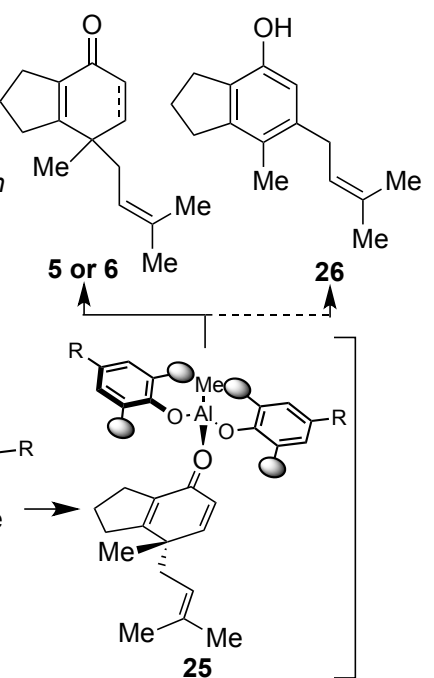
25
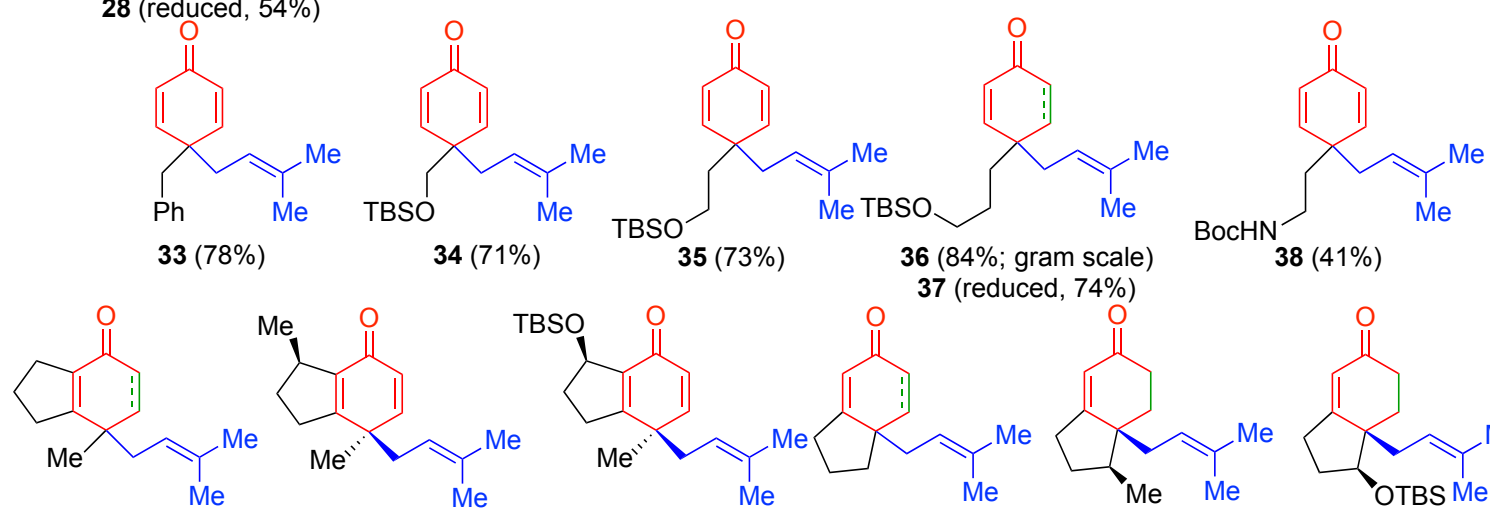

$39(79 \%)$

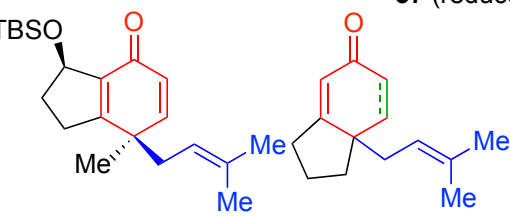

40 (71\%)

7 (78\%)

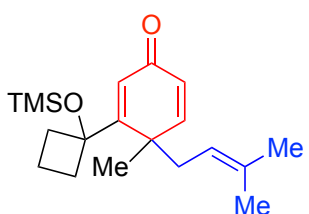

$32(41 \%)$

gram scale)

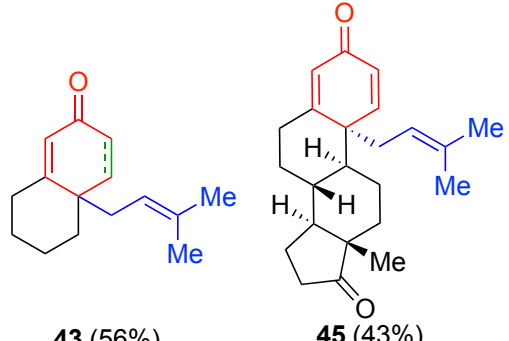

43 (56\%)

$45(43 \%)$

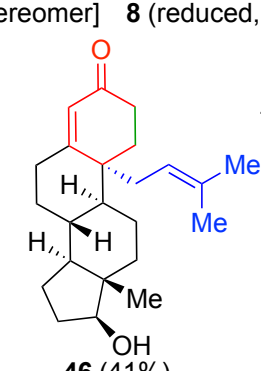

$46(41 \%)$

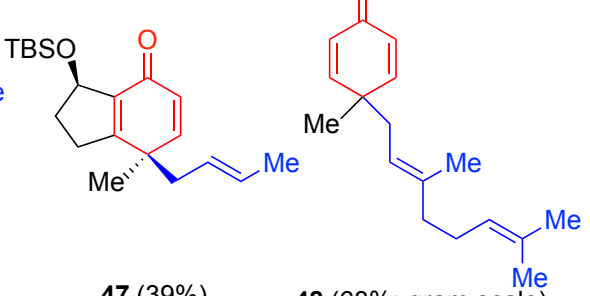

$47(39 \%)$

[single diastereomer]

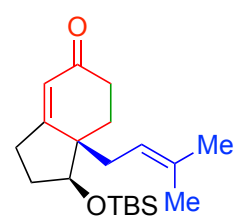

$\mathbf{4 1}$ (69\%; gram scale) $\mathbf{4 2}$ (61\%; gram scale)
[single diastereomer] [single diastereomer]

Figure 2 | Redox neutral and reductive dearomatizations. (a) General process, (b) mechanistic considerations, and (c) overall scope. 

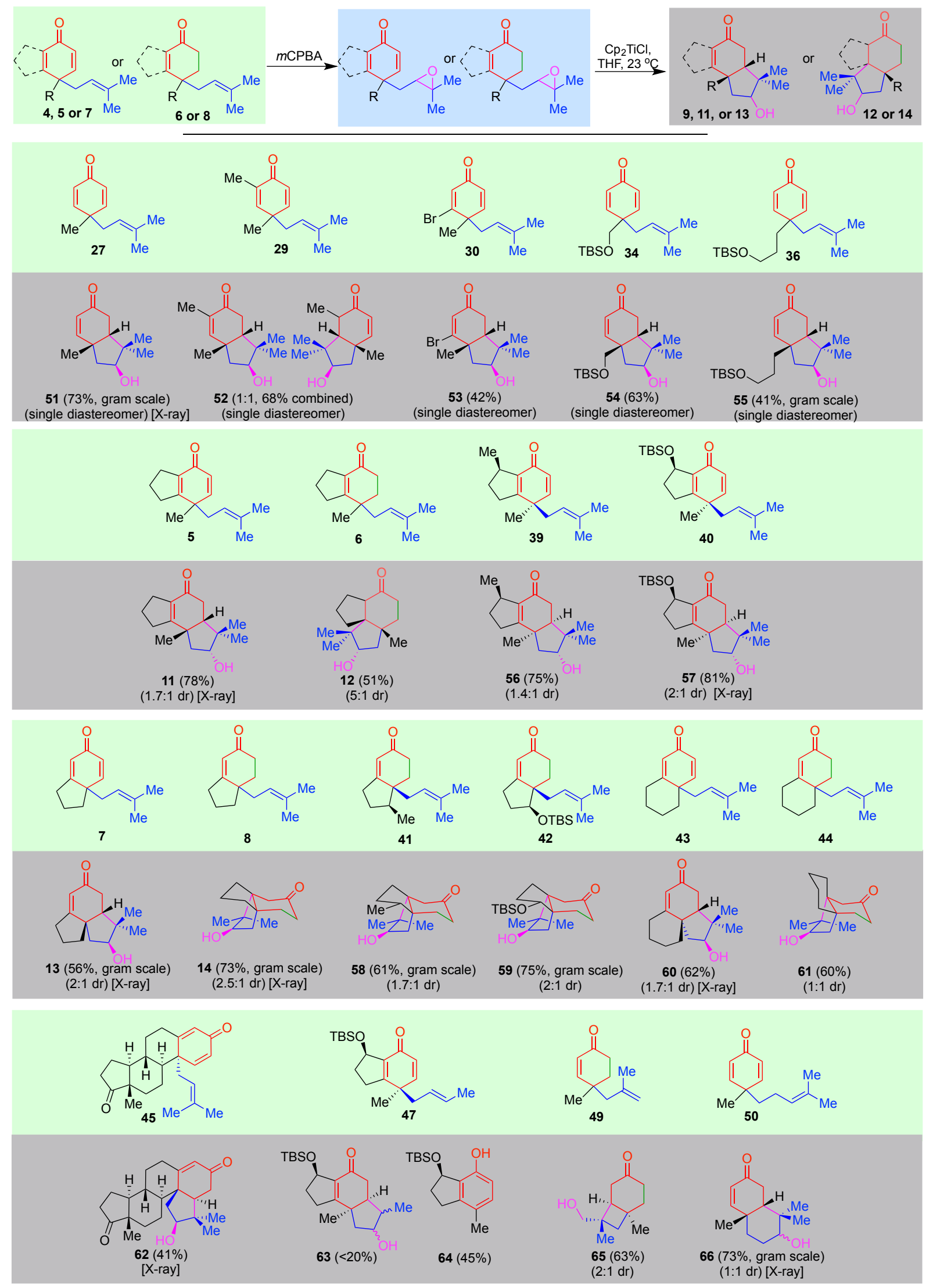

Figure 3 | Scope of epoxidation/radical cyclization cascade. 
a

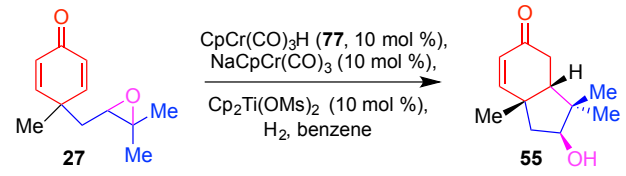

\begin{tabular}{|cccc|}
\hline Entry & Change in Conditions & Solvent & Yield (conversion) \\
\hline 1 & fac-Ir(ppy $)_{3}, \mathrm{Cp}_{2} \mathrm{TiCl}_{2}$, Hantzsch ester & $\mathrm{THF}$ & $<15 \%$ (n.d.) \\
2 & $5 \mathrm{~mol} \% \mathrm{Cp}_{2} \mathrm{TiCl}_{2}, \mathrm{Collidine} \cdot \mathrm{HCl}(2.5 \mathrm{eq})$, & $\mathrm{THF}$ & $40 \%$ (n.d.) \\
3 & $\mathrm{Mn}(1.5 \mathrm{eq}), 2 \mathrm{~d}$ & & \\
3 & $0.1 \mathrm{M}, 70^{\circ} \mathrm{C}, 3 \mathrm{~d}$ & benzene & $47 \%(100 \%)$ \\
4 & $0.1 \mathrm{M}, 50^{\circ} \mathrm{C}, 3 \mathrm{~d}$ & benzene & $11 \%(97 \%)$ \\
5 & $0.1 \mathrm{M}, 50^{\circ} \mathrm{C}, 6 \mathrm{~d}$ & benzene & $22 \%(22 \%)$ \\
6 & $0.02 \mathrm{M}, 70^{\circ} \mathrm{C}, 3 \mathrm{~d}$ & benzene & $85 \%(92 \%)$ \\
7 & $10 \mathrm{~mol} \%\left[\mathrm{Cp}_{2} \mathrm{Ti}(\mu-\mathrm{CO}) \mathrm{Cr}(\mathrm{CO})_{2} \mathrm{Cp}_{2}(76)\right.$ & benzene & $56 \%(69 \%)$ \\
\hline
\end{tabular}

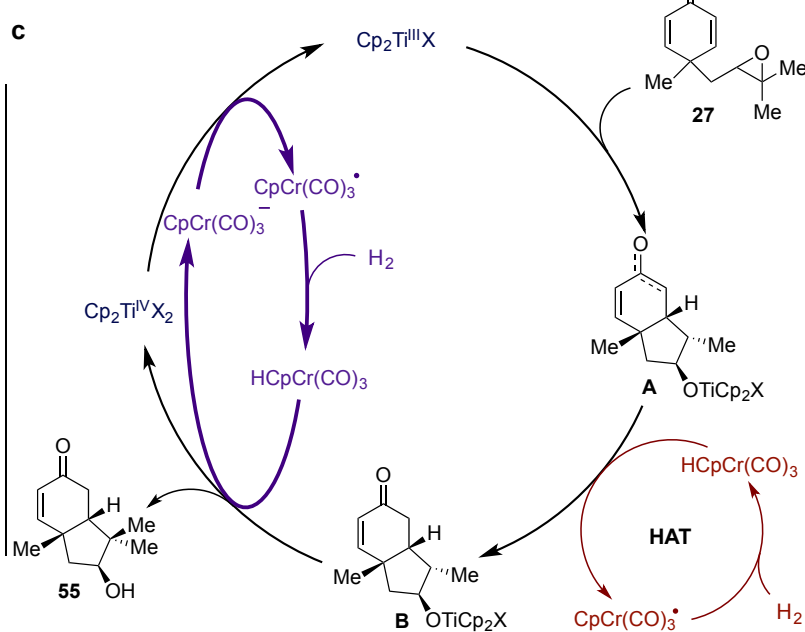

b

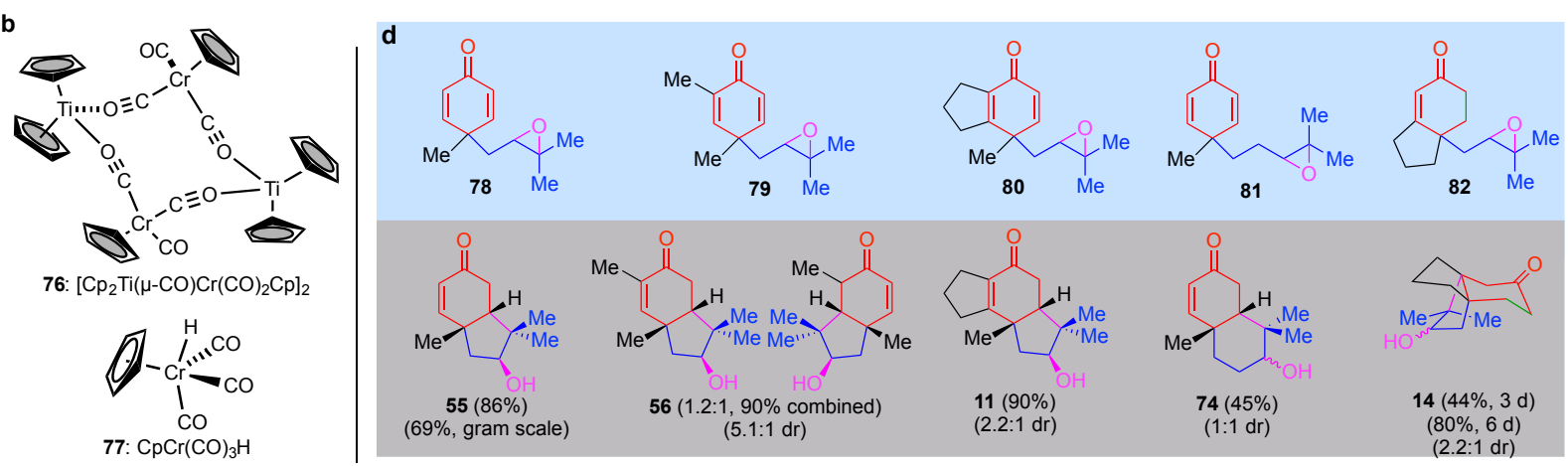

Figure 4 | Exploration of cooperative catalysis to achieve the radical cyalization. (a) Optimization of conditions, (b) structures of metal complexes, (c) proposed mechanism, and (d) scope of the catalytic condition.<smiles>CC1C(O)CC(C)(C)C2C=CC(=O)CC12</smiles>
51 [4 steps]

1) TCDI

23\% AIBN, $n-\mathrm{Bu}_{3} \mathrm{SnH}$ overall) 3) $p$-ABSA

4) $\mathrm{hv}, \mathrm{MeOH}$; DBU

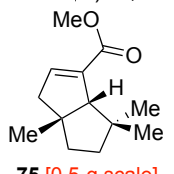

$$
\begin{aligned}
& \text { 1) } \underset{\mathrm{HNMe}(\mathrm{OMe}) \text {, }}{\stackrel{i-\mathrm{PrMgCl}}{2} \mathrm{MeMgBr}} \\
& \text { (88\% overall) }
\end{aligned}
$$

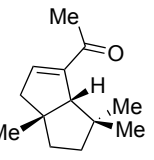

75 [0.5 g scale]

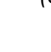

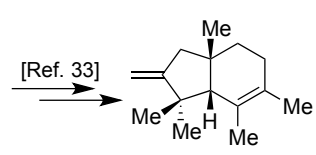

15: cis-preisothapsadiene

$$
\text { This work (5 steps) }
$$
This work (5 steps)
Previous route (10 steps)

ps)

\footnotetext{
$\mathbf{7 6}[0.1 \mathrm{~g}$ scale $]$
This work (10 steps)
Previous route (10 steps)

76 [0.1 g scale $]$
This work (10 steps)
Previous route (10 steps)

76 [0.1 g scale $]$
This work (10 steps)
Previous route (10 steps)
}

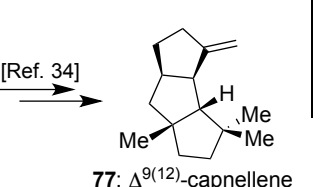

77: $\Delta^{9(12)-c a p n e l l e n e}$

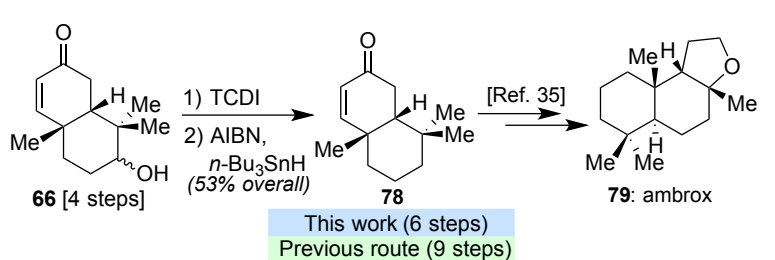

Figure 5| Application of the Radical Cyclization Sequence for Natural Product Synthesis. Use of the developed sequence has led to the expeditious preparation of key intermediates used for the formal total synthesis of four different terpene-based natural products with distinct architectures. 


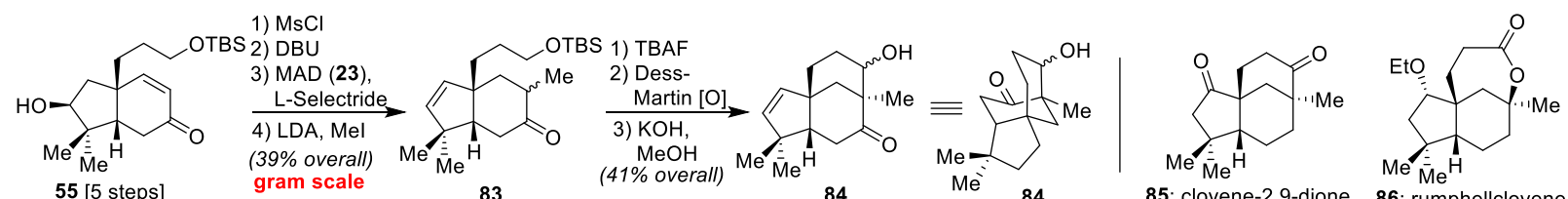

55 [5 steps] gram scale-

85: clovene-2,9-dione 86 : rumphellclovene C
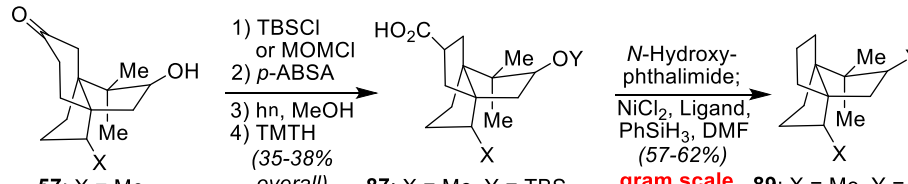

TBAF;

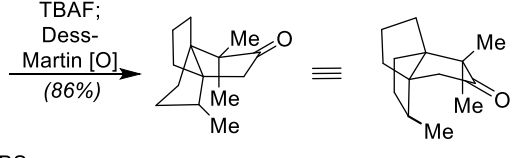
$(35-38 \%$ 7: $X=M e, Y=T B S$ gram scale

58: $X=$ OTBS gram scale $88: X=$ OTBS, $Y=$ MOM

90: $X=$ OTBS, $Y=$ MOM

1) $\mathrm{TBAF}$; Dess-Martin [O] ${ }_{(62 \%)} \mathrm{HCl}$
26

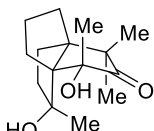

$\mathrm{HO} \mathrm{Me}$

and

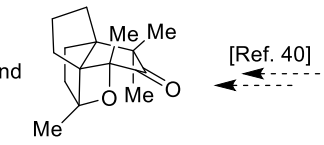

95: dichrocephone B

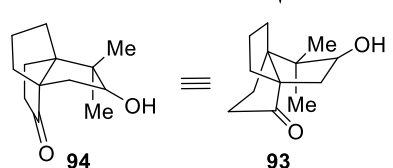

This work (14 steps)

Previous route (20 steps) $\downarrow_{\downarrow}$ [Ref. 39]

96. dichrocephone A

Figure 6 | Further Application of the Radical Cyclization Sequence for Natural Product Synthesis. Use of the developed sequence has led to the expeditious preparation of unique, polycyclic terpene architetures of the clovane, modhephene, and dichrocephone classes. 